\title{
A Parametric Feature Extraction and Classification Strategy for Brain-Computer Interfacing
}

\author{
Dave P. Burke, Member, IEEE, Simon P. Kelly, Member, IEEE, Philip de Chazal, Member, IEEE, \\ Richard B. Reilly, Senior Member, IEEE, and Ciarán Finucane
}

\begin{abstract}
Parametric modeling strategies are explored in conjunction with linear discriminant analysis for use in an electroencephalogram (EEG)-based brain-computer interface (BCI). A left/right self-paced typing exercise is analyzed by extending the usual autoregressive (AR) model for EEG feature extraction with an AR with exogenous input (ARX) model for combined filtering and feature extraction. The ensemble averaged Bereitschaftspotential (an event related potential preceding the onset of movement) forms the exogenous signal input to the ARX model. Based on trials with six subjects, the ARX case of modeling both the signal and noise was found to be considerably more effective than modeling the noise alone (common in BCI systems) with the AR method yielding a classification accuracy of $\mathbf{5 2 . 8} \pm \mathbf{4 . 8 \%}$ and the ARX method an accuracy of $79.1 \pm 3.9 \%$ across subjects. The results suggest a role for ARX-based feature extraction in BCIs based on evoked and event-related potentials.
\end{abstract}

Index Terms-Autoregressive (AR) with exogenous input (ARX), Bereitschaftspotential (BP), brain-computer interface (BCI), electroencephalogram (EEG).

\section{INTRODUCTION}

$\mathbf{F}$ OR SOME people with very severe disabilities (e.g., amyotrophic lateral sclerosis or brainstem stroke), a brain-computer interface (BCI) may be the only feasible channel for communicating with others and for environment control. A BCI typically operates by harnessing signals arising from processes within the brain without depending on the brain's normal output pathways of peripheral nerves or muscles [1]. The most common signal employed for this purpose currently is the noninvasive, scalp recorded electroencephalogram (EEG) [2]-[7]. Complex digital signal processing and pattern recognition techniques are often employed to extract pertinent features from the measured signals in real-time and subsequently used to drive communication and control applications [1].

When designing a BCI, in addition to the technical methodology of recording, feature extraction, and pattern recognition, the choice of feature and elucidation paradigm is paramount. Broadly speaking, most EEG-based BCIs fall into one of four categories: those based on oscillatory EEG components [2], [3]; those based on evoked potentials [4], [5]; those based on slow

Manuscript received December 17, 2002; accepted September 20, 2004.

D. P. Burke was with the Department of Electronic and Electrical Engineering, University College Dublin, Dublin 4, Ireland. He is now with Voxpilot Ltd., Dublin 2, Ireland (e-mail: david.burke@ucd.ie).

S. P. Kelly, P. de Chazal, and R. B. Reilly are with the Department of Electronic and Electrical Engineering, University College Dublin, Dublin 4, Ireland. C. Finucane is with the Department of Medical Physics, St. James' Hospital, Dublin 8, Ireland.

Digital Object Identifier 10.1109/TNSRE.2004.841881 cortical potentials [6]; and, finally, those based on event-related potentials [7]. Autoregressive (AR) models have proved popular in the feature extraction stages of BCIs [8], [9]; see [10] for a review of this method with application to the EEG. The all-pole AR model lends itself well to modeling the EEG as filtered white noise with certain preferred energy bands. The AR with exogenous input (ARX) model is a natural extension to the AR model, facilitating an external input filtered by both AR and moving average components. Cerutti et al. [11] employed the ARX model to filter movement-related brain macropotentials, thereby drastically improving the signal-to-noise ratio of each trial for use in pathophysiological detection. In this paper, we extend the familiar AR feature extraction technique traditionally applied in brain-computer interfacing to the ARX case where an evoked or event-related potential is employed as the exogenous input. The ARX model characterizes the signal into its constituent parts of noise (ongoing EEG) and signal (the ensemble average evoked or event-related potential) [11] and the coefficients of the model constitute the feature vector for subsequent classification.

\section{METHODS}

\section{A. Experiment Paradigm}

A left/right self-paced typing exercise paradigm was employed with subjects sitting upright and fingers in the standard typing position at a Qwerty keyboard. The task consisted of pressing the left or right "home keys" with the corresponding fingers in a self-chosen order and timing. No feedback was given. The EEG was recorded using two bipolar leads with Grass P511 amplifiers (with $50-\mathrm{Hz}$ line filter) from channels $\mathrm{C} 3$ and $\mathrm{C} 4$ of the 10-20 system, corresponding to left and right primary motor areas, respectively. The horizontal electrooculogram (EOG) from the right eye was also recorded. The signals were digitized at a sampling frequency of $512 \mathrm{~Hz}$, low-pass filtered with a cutoff $64 \mathrm{~Hz}$ and down-sampled to 128 samples per second. Six subjects took part in the trials (all male, aged between 22-25 years, in full health) in a single session per subject each lasting $10 \mathrm{~min}$, resulting in a total of $60 \mathrm{~min}$ of data. Key presses were made on average every $2-3$ s resulting in 200-300 epochs of $1500-\mathrm{ms}$ length, ending $120 \mathrm{~ms}$ before the keystroke, thus avoiding effects of EMG activity masquerading as control signals. Trials where significant EOG activity took place (eye blinks) were omitted $(<2 \%$ of the collected data) from analysis (achieved automatically by linearly detrending and removing those time series whose maximum, rectified EOG amplitude exceeded a threshold). 



Fig. 1. Ensemble average of left movements and right movements for electrodes C3 and C4 (movement occurs at $0 \mathrm{~s}$ ). Note the contralateral negativity starting at about 500-ms preceding movement.

\section{B. Bereitschaftspotential $(B P)$}

The experiment paradigm just described produces an event-related potential known as the $\mathrm{BP}$ - a gradually rising negative potential occurring about $1000 \mathrm{~ms}$ preceding the onset of movement [15]. Deecke et al. [16] identified an additional three components of the BP: a pronounced contralateral negativity over the precentral and parietal areas starting about 500-200 ms prior to movement, a small positive deflection beginning around $90 \mathrm{~ms}$ prior to movement, and a smaller negative potential starting about $50 \mathrm{~ms}$ prior to movement predominant over the primary motor cortex. Fig. 1 illustrates the ensemble averages for $\mathrm{C} 3$ and $\mathrm{C} 4$ recordings for left and right self-paced movements from a single subject. Note the contralateral negativity commencing approximately $500 \mathrm{~ms}$ before the onset of movement at $0 \mathrm{~s}$.

\section{Feature Extraction}

To investigate the utility of the ARX method for feature extraction we consider two feature sets for the purposes of comparison.

Case 1: The EEG time series is fitted with an AR model. The AR model can be intuitively rephrased in the frequency domain as white noise source driving an all-pole spectral shaping network $\mathrm{A}^{-1}(\mathrm{z})[10]$. Fig. 2 illustrates the model structure with the

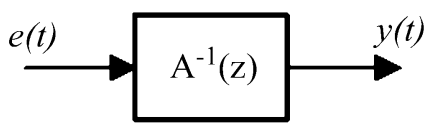

Fig. 2. AR model structure.

output of the model given by (assuming without loss of generality a sampling interval of unity)

$$
\hat{y}(t)=-a_{1} y(t-1)-\cdots-a_{n}\left(t-n_{a}\right)+e(t)
$$

where $e(t)$ is white noise and $a_{i}$ are the coefficients of the $n_{a}$ order model.

Denoting $\theta$ as the vector of model parameters, the forward prediction error is given by

$$
e(t, \theta)=y(t)-\hat{y}(t, \theta)
$$

To fit the model to the data $\mathrm{y}(\mathrm{t})$ collected over a period $t=$ $1, \ldots, N$, we choose such that it minimizes

$$
E(\theta)=\frac{1}{N} \sum_{t=1}^{N} e^{2}(t, \theta) .
$$

Typically, the least squares criterion is employed to find $\hat{\theta}$, resulting in the Yule-Walker equations, which may be solved easily [10]. 


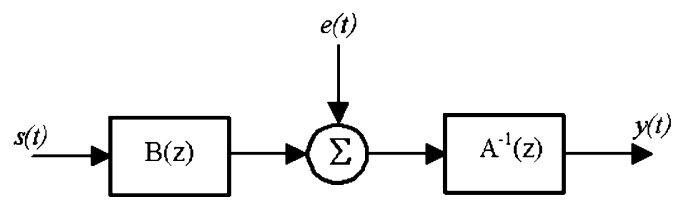

Fig. 3. ARX model structure.

Case 2: Extending the previous case, an ensemble averaged evoked or event-related potential forms the input to an AR moving average (ARMA) model. Assuming a common denominator for the ARMA filter and AR filter results in the ARX model as illustrated in Fig. 3.

Equation (1) is now extended to

$$
\begin{aligned}
\hat{y}(t)= & -a_{1} y(t-1)-\cdots-a_{n_{a}}\left(t-n_{a}\right) \\
& +b_{1} s(t-k)+\cdots+b_{n_{b}}\left(t-k-n_{b}+1\right)+e(t)
\end{aligned}
$$

where $s(t)$ is the exogenous input, $a_{i}$ and $b_{i}$ are the coefficients of the $n_{a}, n_{b}$ order model, $k$ is the delay (which we take as 0 ), and $e(t)$ is white noise. By using (2) and (3), the coefficients of the ARX model can obtained by restating (4) as a linear regression and solving explicitly [12].

In both cases, Akaike's final prediction error (FPE) may be used as a guide for choosing model orders [13]. The FPE penalizes higher order models and is given by

$$
\operatorname{FPE}(\theta)=\frac{N+n_{a}+n_{b}+1}{N-n_{a}-n_{b}-1} E(\theta)
$$

where $n_{b}=0$ for the AR case.

\section{Classification}

A linear discriminant analysis (LDA) was employed for the pattern recognition task in this paper. Consider a set of $c$ classes $C_{k}$. Given an input vector $x$ of features, the classification problem may be formulated in terms of a set of discriminant functions $y_{1}(x), y_{2}(x), \ldots, y_{c}(x)$ where an input vector $\mathrm{x}$ is assigned to class $C_{k}$ if

$$
y_{k}(x)>y_{j}(x), \quad \text { for all } j \neq k
$$

i.e., choose the class for which the corresponding discriminant function is largest. We may derive a discriminant function in terms of Bayes' theorem given by

$$
P\left(C_{k} \mid x\right)=\frac{p\left(x \mid C_{k}\right) P\left(C_{k}\right)}{p(x)} .
$$

Bayes' theorem relates the posterior probability to the product of the class conditional probability and prior probability. The $p(x)$ term serves as a normalization term so that posterior probabilities sum to unity. The usefulness of Bayes' theorem (7) stems from the fact that it is easier to calculate the right-hand side than the left-hand side. One of the simplest discriminant function is $y_{k}=P\left(C_{k} \mid x\right)$ and can be extended further by omitting the class independent probability from (7) and taking the logarithm to obtain

$$
y_{k}(x)=\ln \left(p\left(x \mid C_{k}\right)+\ln \left(P\left(C_{k}\right)\right) .\right.
$$

TABLE I

PARAMETRIC MOdEl ORdERS AND ClassifICATION ACCURACY

\begin{tabular}{l|cccc} 
& $\boldsymbol{n}_{\boldsymbol{a}}$ & $\boldsymbol{n}_{\boldsymbol{b}}$ & ARX acc. \% & AR acc. \% \\
\hline Subject 1 & 4 & 2 & 82.1 & 55.1 \\
Subject 2 & 4 & 2 & 77.8 & 55.0 \\
Subject 3 & 3 & 2 & 79.5 & 60.1 \\
Subject 4 & 4 & 2 & 71.8 & 47.7 \\
Subject 5 & 3 & 2 & 81.1 & 49.1 \\
Subject 6 & 3 & 2 & 82.2 & 49.5 \\
& & Mean: & 79.1 & 52.8
\end{tabular}

An assumption is made that a normal distribution exists for the class conditional densities, given in $d$ dimensions as

$$
p(x)=\frac{1}{(2 \pi)^{d / 2}\left|\Sigma_{k}\right|^{1 / 2}} \exp \left\{-\frac{1}{2}\left(x-\mu_{k}\right)^{T} \Sigma_{k}^{-1}\left(x-\mu_{k}\right)\right\}
$$

where the mean vector and covariance matrix are given, respectively, by

$$
\begin{aligned}
& \mu=E(x) \\
& \Sigma=E\left[(x-\mu)(x-\mu)^{T}\right] .
\end{aligned}
$$

By assuming that covariance matrices are identical for all classes $\Sigma_{k}=\Sigma$, and neglecting constant terms, one forms the linear discriminant function

$$
y_{k}(x)=\mu_{k}^{T} \Sigma^{-1} x-\frac{1}{2} \mu_{k}^{T} \Sigma^{-1} \mu_{k}+\ln \left(P\left(C_{k}\right)\right) .
$$

In case 1, the coefficients of two AR models, one for each channel C3 and C4 were employed as inputs to the LDA classifier. For case 2, the coefficients of four ARX models, (two for each channel with the ensemble average of left movements and right movements as exogenous inputs, respectively) were used as inputs to the classifier.

\section{RESULTS}

To obtain an estimate of accuracy, a cross-validation procedure was employed [14]. The trials for each session (consisting of parametric model coefficients) are first randomly shuffled and subsequently divided into $N$ distinct segments. $N-1$ segments are used to train a LDA classifier and the remaining segment is used as the test set. This process is repeated for each $N$ possible test sets and the mean test set accuracy is computed. Finally, the complete process with a new random shuffle is repeated $M$ times to yield a mean accuracy and standard deviation. The data is uniformly processed with $M=20$ and $N=10$.

Table I displays the LDA classification accuracy for each subject in addition to the parametric model orders that yielded optimum accuracy for the ARX model (the Akaike FPE was used as a starting point for selection of model orders). In all subjects, the optimum classification accuracy was obtained for the same order $n_{a}$ in both the ARX and AR models.

Fig. 4 illustrates the mean accuracy and standard deviation over each shuffle for each subject for the AR and ARX models in the feature extraction stage. The mean accuracy over all subjects for ARX features is $79.1 \pm 3.9 \%$ while for AR features is $52.8 \pm$ 


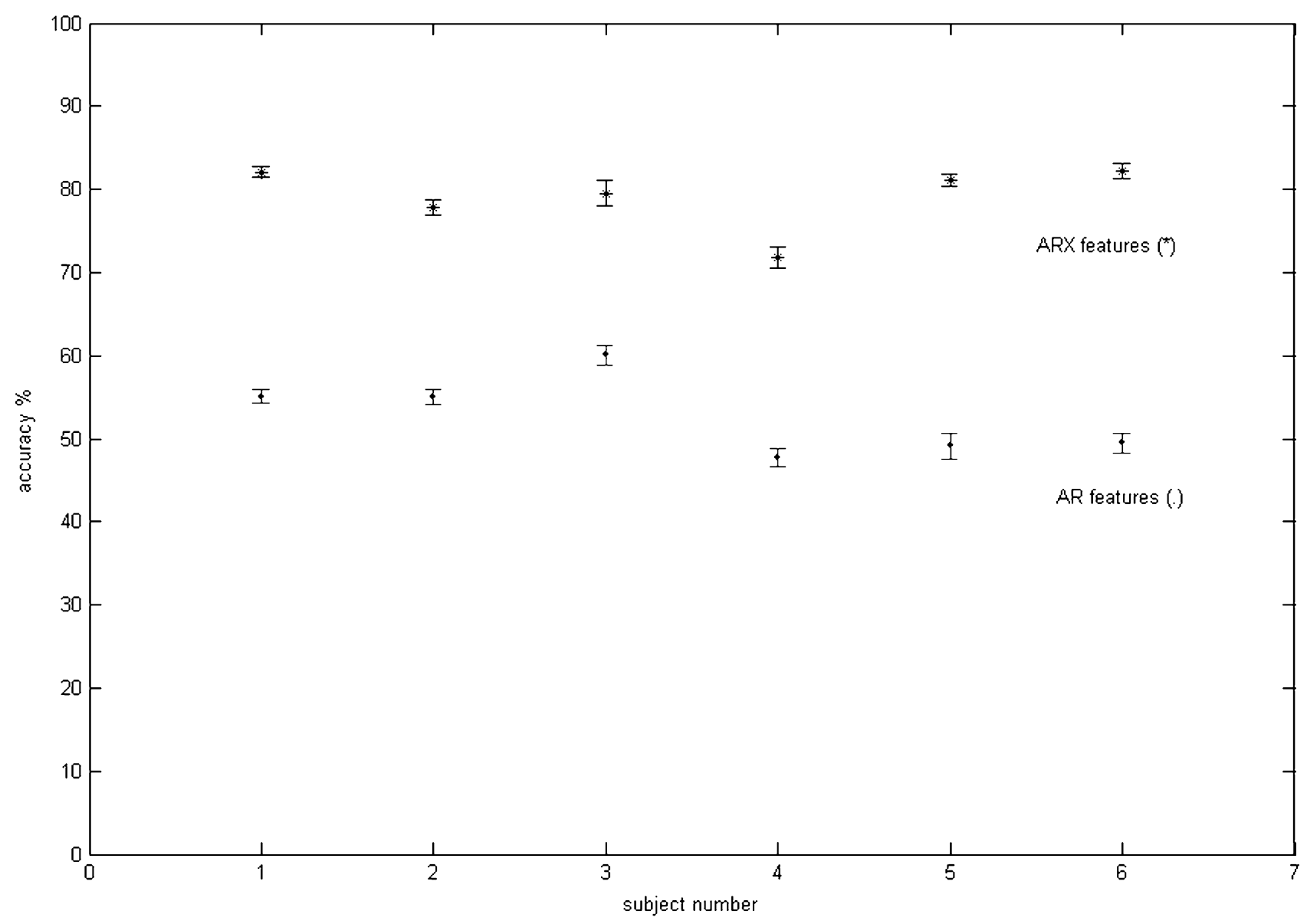

Fig. 4. Mean accuracy $\%$ over $M=20$ random shuffles for each subject for AR and ARX features.

4.8\%. The results show clearly that for each subject the ARX set of features yields higher classification accuracy than the AR features.

\section{DISCUSSION}

The ARX model, while elegant in its simplicity, assumes a linear superposition of the evoked or event-related potential with the background EEG activity. This is a clear simplification of the actual nonlinear interaction expected [11]. The common denominator structure of the ARX model, implying that noise enters early in the modeled process, seems physiological reasonable as ongoing EEG contributions from neighboring neural populations contribute mostly to the background noise [15]. Modeling the contributions of both the known signal (ensemble averaged evoked or event-related potential) and the noise (EEG) yields better features for the classifier and is an intuitively satisfying result.

The ARX model employs an ensemble averaged evoked or event-related potential as its exogenous input. This is valid if the time-locked responses remain identical throughout the session. In practice, responses are never identical and trial-to-trial variability may occur due to a variety of reasons such as sensory adaptation or differing behavioral outcomes [19]. The ensemble average used in conjunction with the ARX model represents a "typical" evoked or event related potential and thus only approximates an individual trial's actual evoked or event related potential.
The experimental paradigm employed short epochs of $1.5 \mathrm{~s}$ for analysis, terminating $120 \mathrm{~ms}$ before the onset of movement. The duration of the epochs is sufficiently short to be considered quasistationary and suitable for parametric modeling [10]. It is likely that longer epochs, for example those used in [3], would yield higher accuracies for the AR model by also extracting pertinent features of event-related desynchronization.

The results suggest that the ARX feature extraction approach should prove useful for BCI systems based on evoked and eventrelated potentials, e.g., [4] and [7]. It is expected that the model orders in (4) for the $b$ coefficients will be larger to correctly model the more complex morphology of P300 and visual evoked potential (VEP) waveforms. Finally, the parametric feature extraction and classification stages are simple to implement, computationally efficient, and thus suitable for implementation in real-time application for BCI systems.

In this paper, an LDA was employed for the classification task. This choice was prompted by the emphasis of the research being on the extraction of pertinent features and not on the classifier itself. We plan to test more sophisticated classification approaches (such as those used in [17]) employed in conjunction with the present ARX feature extraction technique with a view to further improving our classification results.

In this study, we have studied the execution of actual movements as opposed to imagined movements by employing data acquired before the actual movement took place. It has been shown [18] that the BP phenomenon is present to a comparable 
extent in imagined movements albeit with lower amplitudes. We intend to study what effect, if any, imagined movements versus actual movements may have on our results.

\section{CONCLUSION}

The approach of employing an AR model for feature extraction with the EEG is well known [10] with a number of successful applications in BCI systems [8], [9]. The ARX model, by combining information about the underlying event-related $\mathrm{BP}$, performs better than the simple AR model of equivalent order and suggests the role of this approach in BCIs harnessing event-related or evoked potentials.

\section{REFERENCES}

[1] J. R. Wolpaw, N. Birbaumer, W. J. Heetderks, D. J. McFarland, P. H. Peckham, G. Schalk, E. Donchin, L. A. Quatrano, C. J. Robinson, and T. M. Vaughan, "Brain-computer interface technology: A review of the first international meeting," IEEE Trans. Rehab. Eng., vol. 8, no. 2, pp. 164-173, Jun. 2000.

[2] J. R. Wolpaw, D. J. McFarland, G. W. Neat, and C. A. Forneris, "An EEG-based brain-computer interface for cursor control," Electroencephalogr. Clin. Neurophysiol., vol. 78, pp. 252-259, 1991.

[3] G. Pfurtscheller, J. Kalcher, C. Neuper, D. Flotzinger, and M. Pregenzer, "On-line EEG classification during externally-paced hand movements using a neural network-based classifier," Electroencephalogr. Clin. Neurophysiol., vol. 99, pp. 416-425, 1996.

[4] E. Sutter, "The brain response interface: Communication through visually-induced electrical brain response," J. Microcomput. Appl., vol. 15, pp. 31-45, 1992.

[5] M. Middendorf, G. McMillan, G. Calhoun, and K. S. Jones, "Brain-computer interfaces based on the steady-state visual-evoked response," IEEE Trans. Rehab. Eng., vol. 8, no. 2, pp. 211-214, Jun. 2000.

[6] N. Birbaumer, N. Ghanayim, T. Hinterberger, I. Iversen, B. Kotchoubey, A. Kuebler, J. Perelmouter, E. Taub, and H. Flor, "A spelling device for the completely paralyzed," Nature, vol. 398, pp. 297-298, 1999.

[7] L. A. Farwell and E. Donchin, "Talking off the top of your head: A mental prosthesis utilizing event-related brain potentials," Electroencephalogr. Clin. Neurophysiol., vol. 70, pp. 355-372, 1988.

[8] W. D. Penny, S. J. Roberts, E. A. Curran, and M. J. Stokes, "EEG-based communication: A pattern recognition approach," IEEE Trans. Rehab. Eng., vol. 8, pp. 214-216, June 2000.

[9] C. Guger, A. Schlögl, C. Neuper, D. Walterspacher, T. Strein, and G. Pfurtscheller, "Rapid prototyping of an EEG-based brain-computer interface (BCI)," IEEE Trans. Rehab. Eng., vol. 9, no. 1, pp. 49-58, Mar. 2001.

[10] J. Pardey, S. Roberts, and L. Tarassenko, "A review of parametric modeling techniques for EEG analysis," Med. Eng. Phys., vol. 18, pp. 2-11, 1995.

[11] S. Cerutti, G. Chiarenza, D. Liberati, P. Mascellani, and G. Pavesi, "A parametric method of identification of single-trial event-related potentials in the brain," IEEE Trans. Biomed. Eng., vol. 35, no. 9, pp. 701-711, Sep. 1988.

[12] L. Ljung, System Identification: Theory for the User. Englewood Cliffs, NJ: Prentice-Hall, 1987.

[13] H. Akaike, "Fitting autoregressions for prediction," Ann. Inst. Stat. Math., vol. 21, pp. 243-247, 1969.

[14] C. M. Bishop, Neural Networks for Pattern Recognition. Oxford, U.K.: Clarendon, 1995.

[15] K. E. Misulis, Spehlmann's Evoked Potential Primer: Visual, Auditory, and Somatosensory Evoked Potentials in Clinical Diagnosis. Burlington, MA: Butterworth-Heinemann, 1994.

[16] L. Deecke, R. P. Scheid, and H. H. Kornhuber, "Distribution of readiness potential, pre-motion positivity and motor potential of the human cerebral cortex preceding voluntary finger movement," Exp. Brain Res., vol. 7, pp. 158-168.
[17] B. Blankertz, G. Curio, and K.-R. Müller, "Classifying single trial EEG: Toward brain computer interfacing," presented at the 16th Annu. Conf. Neural Information Processing Systems (NIPS), Vancouver, BC, Canada, 2002.

[18] R. Beisteiner, P. Hollinger, G. Lindinger, W. Lang, and A. Berthoz, "Mental representations of movements. Brain potentials associated with imagination of hand movements," Electroencephalogr. Clin. Neurophysiol., vol. 96, pp. 183-193, 1995.

[19] D. H. Lange and G. F. Inbar, "Modern techniques in ERP research'," in Modern Techniques in Neuroscience Research, U. Windhorst and H. Johansson, Eds. Berlin, Germany: Springer-Verlag, 1999.

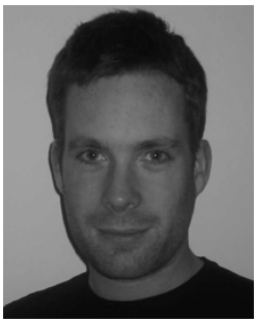

Dave P. Burke (M'04) received the B.E., M.Eng.Sc., and $\mathrm{Ph} . \mathrm{D}$. degrees in electronic engineering from University College Dublin, Dublin, Ireland, in 1997, 1999, and 2003, respectively.

From 1999 to 2001, he was a Research Coordinator with the Rehabilitation Engineering Laboratory, National Rehabilitation Hospital, Dublin, where he specialized in dynamical systems modeling of the EEG and biomedical signal processing. In 2000, he was involved in founding Voxpilot Ltd, Dublin, where he currently serves as Chief Technology Officer. He is actively involved in the design of next-generation Internet systems and is an Editor and Contributor to World Wide Web Consortium and Internet Engineering Task Force specifications.

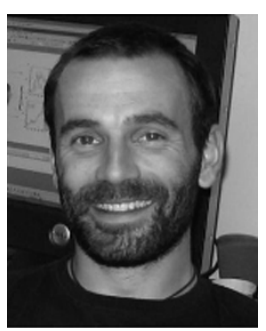

Simon P. Kelly (M'04) received the B.E. degree in electronic engineering, in 2001, from University College Dublin, Dublin, Ireland, where he is currently working toward the Ph.D. degree in electronic and electrical engineering.

His current research is centered on the human attention system, studied from both a cognitive neurophysiology and rehabilitation engineering perspective.

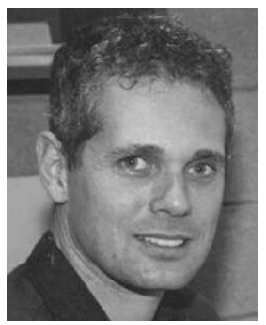

Philip de Chazal (M'94) received the B.E. degree in electronic engineering, and the M.Biomed.E. and $\mathrm{Ph}$.D. degrees in biomedical engineering from University of New South Wales, Sydney, Australia, in 1989, 1995, and 1999 respectively.

$\mathrm{He}$ was a Research Scientist for the CSIRO, Sydney, from 1990 to 1992, a Biomedical Engineer for MedCare Systems, Sydney from 1993 to 1999 and a Research Fellow at University College Dublin, Dublin, Ireland, from 1999 to 2003. He is cofounder and currently the Chief Technical Officer of BiancaMed, Dublin, Ireland, a company providing intelligent computer-based analysis of medical signals. His research interests include signal processing and pattern recognition for biomedical applications and image processing for multimodal applications.

Dr. de Chazal is a member of the IEEE Engineering in Medicine and Biology Society. He is a reviewer for the IEEE TRANSACTIONS ON MULTIMEDIA and the IEEE TRANSACTIONS ON BIOMEDICAL ENGINEERING. 


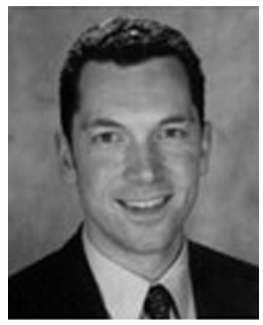

Richard B. Reilly (M'92-SM'04) received the B.E., M.Eng.Sc., and Ph.D. degrees (all in electronic engineering) from the National University of Ireland, Dublin, in 1987, 1989, and 1992, respectively.

In 1988, he joined Space Technology Ireland, Maynooth, Ireland, and the Department de Recherche Spatiale (CNRS Group), Paris, France, where he was developing DSP-based on-board experimentation for the NASA satellite WIND. In 1990, he joined the National Rehabilitation Hospital, Dublin, and in 1992 became a Postdoctoral Research Fellow with the University College Dublin, focusing on signal processing for speech and gesture recognition. Since 1996, he has been a member of the Academic Staff of the Department of Electronic and Electrical Engineering, University College Dublin. He is currently a Senior Lecturer. He is currently an Associate Editor for the Journal of Applied Signal Processing, Signal Processing and the IEE Proceedings Vision, Image \& Signal Processing. His research interests include neurological signal processing and multimodal signal processing.

Dr. Reilly was the 1999/2001 Silvanus P. Thompson International Lecturer for the IEE. In 2004, he was awarded a U.S. Fulbright Award for research collaboration into multisensory integration with the Nathan Kline Institute for Psychiatric Research, New York. He is a member of the IEEE Engineering in Medicine and Biology Society and Signal Processing Society. He is the Republic of Ireland representative on the Executive Committee of the IEEE United Kingdom and Republic of Ireland Section. He is an Associate Editor for the IEEE TRANSACTIONS ON MULTIMEDIA and also a reviewer for the IEEE TRANSACTIONS ON BIOMEDICAL ENGINEERING, the IEEE TRANSACTIONS on Neural Systems and Rehabilitation Engineering, and the IEEE TRANSACTIONS ON INDUSTRIAL ELECTRONICS.
Ciarán Finucane received the B.E. degree in electronic engineering from the University College Dublin (UCD), Dublin, Ireland, in 2001, and the M.S. degree in engineering science from UCD and the National Rehabilitation Hospital, Dublin, for work entitled "EEG based Brain Computer Interfaces for the Disabled" in 2003.

In 2003, he joined the Department of Medical Physics, St. James' Hospital Dublin, where he is currently a Medical Physcist. His research interests include the development of novel BCIs, neurophysiological signal analysis, biomedical applications of multimedia, wireless and Internet technologies, and biological systems modeling. 